OPEN ACCESS

Edited by: Ashutosh Goel,

Rutgers, The State University of New Jersey, USA

Reviewed by: Minoru Tomozawa,

Rensselaer Polytechnic Institute, USA Prabhat K. Gupta,

The Ohio State University, USA Jeetendra Sehgal,

Sterlite Technologies Limited, India Yaqoot Shaharyar,

Rutgers, The State University of New Jersey, USA

${ }^{*}$ Correspondence: Yuanzheng Yue, Fredrik Bajers Vej $7 \mathrm{H}$, Aalborg, Denmark yy@bio.aau.dk

Specialty section: This article was submitted to Glass Science, a section of the journal Frontiers in Materials

Received: 08 April 2015 Accepted: 15 July 2015 Published: 07 August 2015

Citation:

Yue Y (2015) Anomalous enthalpy relaxation in vitreous silica. Front. Mater. 2:54. doi: 10.3389/fmats.2015.00054

\section{Anomalous enthalpy relaxation in vitreous silica}

\author{
Yuanzheng Yue ${ }^{1,2 *}$ \\ 'State Key Laboratory of Silicate Materials for Architectures, Wuhan University of Technology, Wuhan, China, ${ }^{2}$ Department \\ of Chemistry and Bioscience, Aalborg University, Aalborg, Denmark
}

It is a challenge to calorimetrically determine the glass transition temperature $\left(T_{\mathrm{g}}\right)$ of vitreous silica. Here, we demonstrate that this challenge mainly arises from the extremely high sensitivity of the $T_{\mathrm{g}}$ to the hydroxyl content, but also from decrease of the $T_{\mathrm{g}}$ with repeating the calorimetric scans. It is known that the liquid fragility (i.e., the speed of the viscous slow-down of a supercooled liquid at its $T_{\mathrm{g}}$ during cooling) has impact on enthalpy relaxation in glass. Here, we find that vitreous silica (as a strong system) exhibits striking anomalies in both glass transition and enthalpy relaxation compared to fragile oxide systems. The anomalous enthalpy relaxation of vitreous silica is discovered by performing the hyperquenching-annealing-calorimetry experiments. We argue that the strong systems like vitreous silica and vitreous Germania relax in a structurally cooperative manner, whereas the fragile ones do in a structurally independent fashion. We discuss the origin of the anomalous enthalpy relaxation in the $\mathrm{HQ}$ vitreous silica.

Keywords: vitreous silica, glass transition temperature, enthalpy relaxation, hyperquenching, annealing

\section{Introduction}

Vitreous silica is of special importance for advanced technology, e.g., for communication and semiconductor technologies, due to its excellent mechanical, thermal, and optical properties simultaneously connected with excellent chemical resistance (Bateson, 1958; Thomas, 1958; Brückner, 1971; Doremus, 2002; Mei et al., 2007; Lascaris et al., 2014). Despite its simple chemical identity, vitreous silica is one of the most interesting and complicated glass formers regarding its structure, dynamics, and properties. In a review paper (Brückner, 1971), Brückner has described physical anomalies of vitreous silica with regard to the volume relaxation, thermal expansion, specific heat capacity, and transport phenomena. The diffusion and viscous flow in silica have been discussed by Doremus (2002). Anomalous behaviors of liquid silica have also been found, e.g., the fragile-to-strong liquid transition and the polyamorphism in the energy landscape (Saika-Voivod et al., 2001), liquid-liquid critical point (Lascaris et al., 2014). Recently, the role of the structural disorder in the thermodynamics and atomic dynamics of glasses has been revealed by comparing the atomic vibrational dynamics of compressed vitreous silica with that of various kinds of corresponding crystals (Chumakov et al., 2014). Despite progress in understanding anomalous physical behaviors of vitreous silica, some crucial questions still remain open. For instance, what is the calorimetric glass transition temperature $\left(T_{\mathrm{g}}\right)$ of a perfectly pure vitreous silica? Why does the $T_{\mathrm{g}}$ of vitreous silica vary with the number of calorimetric scans in contrast to that of other glass formers? How can one get the true $T_{\mathrm{g}}$ of pure vitreous silica? How does the enthalpy of hyperquenched (HQ) vitreous silica evolve during sub- $T_{\mathrm{g}}$ annealing? In this paper we attempt to answer these questions, at least to some extent.

Calorimetric studies of enthalpy relaxation in HQ glasses (e.g., those cooled at $>10^{4} \mathrm{~K} / \mathrm{s}$ ) is helpful for understanding the nature of glass and glass transition (Huang and Gupta, 1992; Yue, 2002; 
Yue et al., 2002a,b; Angell et al., 2003; Yue and Angell, 2004; Monaco et al., 2006; Hornbøll and Yue, 2008a; Hu et al., 2011). Such studies have provided insights into the energetic and structural heterogeneities in supercooled liquids and their glass states (Yue et al., 2002a; Zhang et al., 2013a,b; Zhou et al., 2015), which would be difficult to be attained by other means. The principle of the experimental approach of these studies is to trap the high potential energy state of a liquid by hyperquenching, and subsequently discharge the excessive energy by annealing at a certain temperature $\left(T_{\mathrm{a}}\right)$ below $T_{\mathrm{g}}$ for a certain period of time $\left(t_{\mathrm{a}}\right)$, and then upscanning the annealed HQG in a differential scanning calorimeter (DSC). Liquid structure can be frozen-in by hyperquenching and thereby a higher fictive temperature $\left(T_{\mathrm{f}}\right)$ compared to $T_{\mathrm{g}}$ will be generated in glass state (Yue et al., 2002b; Kiczenski and Stebbins, 2006; Mauro et al., 2009a). With proceeding of the relaxation process, the $T_{\mathrm{f}}$ gradually decreases with increasing $T_{\mathrm{a}}$ and $t_{\mathrm{a}}$. By annealing, we observe a gradual shift of enthalpy release peak of the calorimetric upscan curve toward higher temperatures, implying that there is a distribution of configurational states of the liquid at $T_{\mathrm{f}}$. This also indicates that the liquid at $T=T_{\mathrm{f}}$ is energetically and structurally heterogeneous (Yue and Angell, 2004). The direct comparative studies about the enthalpy relaxation and the evolution of the vibrational density of states in HQ glasses during annealing has enhanced the understanding of the atomic vibrational dynamics in glass from different angles (Angell et al., 2003; Monaco et al., 2006).

According to previous reports (Hornbøll and Yue, 2008b; Mauro and Loucks, 2008), the liquid fragility has an impact on the enthalpy relaxation in glass. Numerous experimental and modeling studies have been done on the enthalpy relaxation of HQ fragile oxide systems (Huang and Gupta, 1992; Yue, 2002, 2004; Yue et al., 2002a,b; Angell et al., 2003; Guo et al., 2012; Zhang et al., 2013a,b,c), but to our best knowledge, almost no investigations have been conducted on the HQ prototypical strong glass former - HQ vitreous silica. It is expected that the enthalpy relaxation in HQ vitreous silica differs from HQ fragile glass formers owing to their structural differences in terms of both short-range and medium-range order. The former one has well-developed structure units with a higher degree of short-range order, covalence-dominant bonds, and higher network connectivity, whereas the former ones possess less well-developed structure units with a lower degree of shortrange order, more ionic bonds, and lower network connectivity. It should be noted that the liquid fragility mentioned here refers to the dynamic nature of a glass forming liquid, i.e., the viscous slow-down speed of a supercooled liquid at $T_{\mathrm{g}}$ upon cooling, and is physically different from the mechanical fragility of a glass. Concerning the relaxation behavior of the strong system - vitreous silica, some experimental results have been reported, which deal with, e.g., surface relaxation (Agarwal and Tomozawa, 1997; Koike and Tomozawa, 2006), structural anisotropy relaxation, and volume relaxation (Murach and Brückner, 1997), but no results deal with the enthalpy relaxation of the HQ vitreous silica. In this work, we focus on the enthalpy response of the HQ vitreous silica $\left(\mathrm{HQSiO}_{2}\right)$ to sub- $T_{\mathrm{g}}$ annealing, and compare it with that of the HQ fragile oxide systems such as HQ basaltic glass (HQBas) (Yue and Angell, 2004) and calcium metaphosphate glass (HQCmP) (Yue, 2002), and also with that of the $\mathrm{HQ}$ strong vitreous $\mathrm{GeO}_{2}\left(\mathrm{HQGeO}_{2}\right)$ (Hornbøll and Yue, 2008b).

In this paper, we provide the experimental evidence for the striking differences in enthalpy relaxation between strong and fragile glass systems by performing hyperquenchingannealing-calorimetry experiments on two strong systems and two fragile systems. We explore the anomalous enthalpy relaxation behavior of $\mathrm{HQSiO}_{2}$ and discuss its origin in terms of the potential energy landscape. To identify the universal feature of the enthalpy relaxation of strong glass formers, we compare the relaxation of $\mathrm{HQSiO}_{2}$ with that of $\mathrm{HQGeO}_{2}$ (Hornbøll and Yue, 2008b; Hu and Yue, 2008). Besides its fundamental importance, the investigation of the enthalpy relaxation in HQ strong glass formers is also of technological significance, since the annealing process has strong impact on the physical performances of those glasses. Such studies could give insight into the physical origin of liquid fragility.

\section{Experimental}

\section{Samples}

The bulk vitreous silica used for the $T_{\mathrm{g}}$ study was obtained from Heraeus Holding $\mathrm{GmbH}$. The sample was produced by using electric fusion process, which enabled the hydroxyl content to be $<1 \mathrm{ppm}$. The thus-prepared vitreous silica contains total metallic impurities below $\sim 20 \mathrm{ppm}$. The impurities consist primarily of $\mathrm{Al}_{2} \mathrm{O}_{3}$ with much smaller amounts of alkali oxide, $\mathrm{Fe}_{2} \mathrm{O}_{3}$, $\mathrm{TiO}_{2}, \mathrm{MgO}$, and $\mathrm{ZrO}_{2}$. These impurities play a role primarily as network modifiers that depolymerize the structural network of glass. The vitreous silica used for the viscosity measurements of Urbain et al. (1982) contains $<10$ ppm hydroxyl and $<60$ ppm metallic impurities.

The HQ vitreous silica $\left(\mathrm{HQSiO}_{2}\right)$ samples used for the relaxation study were obtained from Technical University of Berlin, which were the continuous fibers with an average diameter of $8.5 \mu \mathrm{m}$ and a hydroxyl content of $1021 \mathrm{ppm}$ (Murach and Brückner, 1997). The fibers were drawn from a cylindrical preform of vitreous silica (diameter: $8 \mathrm{~mm}$ ) at $2703 \mathrm{~K}$ (Murach and Brückner, 1997). The vitreous silica preform was fabricated by Heraeus Holding $\mathrm{GmbH}$ by means of a synthetic process. The thus-derived vitreous silica contains a much lower amount of metallic impurities $(<1 \mathrm{ppm})$, compared to the vitreous silica obtained by a fusion process.

Both $\mathrm{HQ} \mathrm{GeO}_{2}$ glass $\left(\mathrm{HQGeO}_{2}\right.$ ) and $\mathrm{HQ} \mathrm{CaP}_{2} \mathrm{O}_{6}$ glass (i.e., $\mathrm{HQ}$ calcium metaphosphate - HQCmP) were produced as continuous fibers using the crucible technique (Yue, 2002; Hornbøll and Yue, 2008b). The HQ basalt (HQBas) was produced as wool fibers by using the centrifugal force cascade process (Yue et al., 2002a) and provided by Rockwool International A/S. The chemical composition (mol\%) of $\mathrm{HQBas}$ is $47.5 \mathrm{SiO}_{2}, 9.1 \mathrm{Al}_{2} \mathrm{O}_{3}$, 6.6 FeO, 15.9 $\mathrm{CaO}, 16.9 \mathrm{MgO}, 2.0 \mathrm{Na}_{2} \mathrm{O}, 0.7 \mathrm{~K}_{2} \mathrm{O}$. The cooling rates of all the fiber samples were estimated using our previously established method (Yue et al., 2004). The characteristic parameters (such as $T_{\mathrm{g}}$, average fiber diameter, approximate cooling rate) and preparation methods of the four kinds of fiber samples $\left(\mathrm{HQSiO}_{2}, \mathrm{HQGeO}_{2}, \mathrm{HQBas}\right.$, and $\mathrm{HQCmP}$ ) are listed in Table 1. Note that $\mathrm{HQGeO}_{2}, \mathrm{HQBas}$, and HQCmP contains hydroxyl 
TABLE 1 | Characteristic parameters and preparation methods of the HQ glass fibers.

\begin{tabular}{lrrrrcrl} 
Fiber & $\boldsymbol{T}_{\mathbf{g}}(\mathbf{K})$ & $\boldsymbol{T}_{\mathbf{f}}(\mathbf{K})$ & $\boldsymbol{T}_{\mathbf{f}} / \boldsymbol{T}_{\mathbf{g}}$ & $\boldsymbol{m}$ & $\begin{array}{c}\text { Diameter } \\
(\boldsymbol{\mu} \mathbf{m})\end{array}$ & $\begin{array}{c}\text { Cooling } \\
\text { rate (K/s) }\end{array}$ & $\begin{array}{c}\text { Fiberizing } \\
\text { method }\end{array}$ \\
\hline $\mathrm{HQSiO}_{2}$ & 1336 & 2247 & 1.60 & 20 & $\sim 8.5$ & $\sim 10^{5.5}$ & Preform \\
$\mathrm{HQGeO}_{2}$ & 830 & 1271 & 1.53 & 18 & $\sim 30$ & $\sim 10^{5}$ & Crucible \\
$\mathrm{HQBaS}$ & 941 & 1155 & 1.23 & 44 & $\sim 4.0$ & $\sim 10^{6}$ & Cascade \\
$\mathrm{HQCmP}$ & 803 & 846 & 1.05 & 69 & $\sim 110$ & $\sim 10^{4}$ & Crucible
\end{tabular}

The $T_{g}$ values (error range: $\pm 1 \mathrm{~K}$ ) were determined from the second DSC upscan curves on the fiber samples relaxed during the first DSC scans. The $T_{f}$ values (error range: $\pm 5 K$ ) were determined from the as-HQ sample using the method proposed elsewhere (Yue et al., 2002a). The fragility indices $(m)$ were determined from the viscosity curves shown in Figure 3.

group $(<300 \mathrm{ppm})$. The $T_{\mathrm{g}}$ values of fragile glass systems also drops with increasing hydroxyl content (Deubener et al., 2003), but in the range of low hydroxyl content they are much less sensitive to hydroxyl than those of vitreous silica.

\section{Differential Scanning Calorimetry}

The isobaric heat capacities $\left(C_{\mathrm{p}}\right)$ of both bulk and HQ fiber vitreous silica samples were measured by the present author by using the differential scanning calorimetry (DSC) (Netzsch STA 449C Jupiter). To determine the $C_{\mathrm{p}}$ curves of the samples, both the baseline (blank) and the reference sample (sapphire) were measured. The samples for the enthalpy relaxation studies were upscanned in the DSC in argon from $333 \mathrm{~K}$ to a temperature above $T_{\mathrm{g}}$ at $20 \mathrm{~K} / \mathrm{min}$ and subsequently downscanned to room temperature $(298 \mathrm{~K})$ at the same rate. Using the same procedure, they were then scanned in DSC for the second time. The $T_{\mathrm{g}}$ values were determined as the onset temperatures of glass transition from the $C_{\mathrm{p}}$ curve of the second DSC upscan. $T_{\mathrm{g}}$ is assigned as the temperature at the cross point between the extrapolated line of the glass $C_{\mathrm{p}}$ and the line of the rapidly rising $C_{\mathrm{p}}$. The thusobtained $T_{\mathrm{g}}$ values are in a good agreement with that determined by the enthalpy-matching method (Moynihan et al., 1976; Yue et al., 2002a; Yue, 2008). The $C_{\mathrm{p}}$ curves of $\mathrm{HQGeO}_{2}, \mathrm{HQBas}$, and HQCmP were adopted from the literature (Yue, 2002; Yue et al., 2002a; Hornbøll and Yue, 2008b, respectively), which were used for studying the differences in enthalpy relaxation between the fragile and the strong glass systems (particularly vitreous silica). The $T_{\mathrm{g}}$ values of $\mathrm{GeO}_{2}$, basalt, and $\mathrm{CaP}_{2} \mathrm{O}_{6}$ glasses were measured using DSC on bulk samples at $10 \mathrm{~K} / \mathrm{min}$ equal to the first downscan rate. The $T_{\mathrm{g}}$ of vitreous silica was measured at $20 \mathrm{~K} / \mathrm{min}$ equal to the first downscan rate (see Figures 1 and 2). The fictive temperatures $\left(T_{\mathrm{f}}\right)$ were determined using the method proposed by Yue et al. (2002), and are listed in Table 1 . The $T_{\mathrm{f}} / T_{\mathrm{g}}$ ratios are also given in Table 1.

Here, it is important to stress that the $T_{\mathrm{g}}$ values of vitreous silica shown in this paper refer to the onset temperatures of the calorimetric glass transition, but are not the true $T_{\mathrm{g}}$ (instead, apparent $T_{\mathrm{g}}$ ), since the samples contain hydroxyl (Figure 1) and were subjected to repeated DSC scans (Figure 2).

\section{Viscosity and Liquid Fragility}

The viscosity data of silica (with $<10 \mathrm{ppm}$ hydroxyl and a $T_{\mathrm{g}}$ value of $1406 \mathrm{~K}$ ) were adopted from the literature (Urbain et al.,

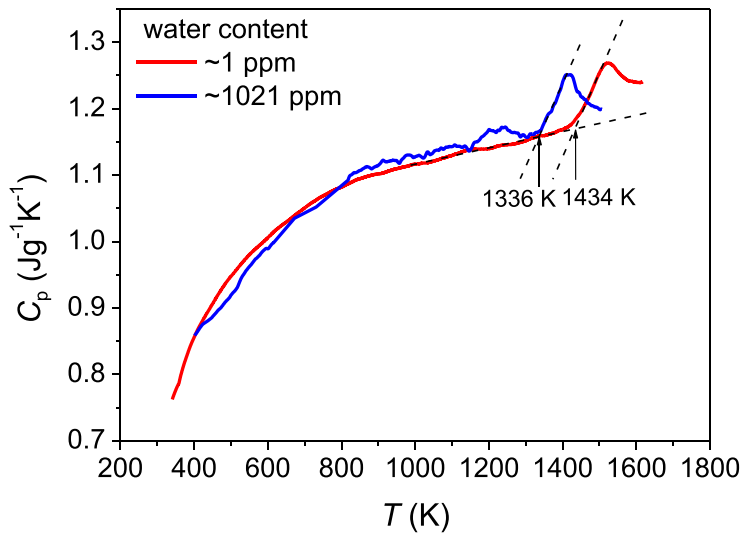

FIGURE 1 | Isobaric heat capacity $\left(C_{p}\right)$ measured during the second DSC upscan as a function of temperature $(T)$ for both the bulk vitreous silica with $\sim 1 \mathrm{ppm}$ hydroxyl (red line) and the vitreous silica fibers $\left(\mathrm{HQSiO}_{2}\right.$ ) with 1021 ppm hydroxyl (blue line). The DSC upscan rate is equal to the prior downscan rate of $20 \mathrm{~K} / \mathrm{min}$.

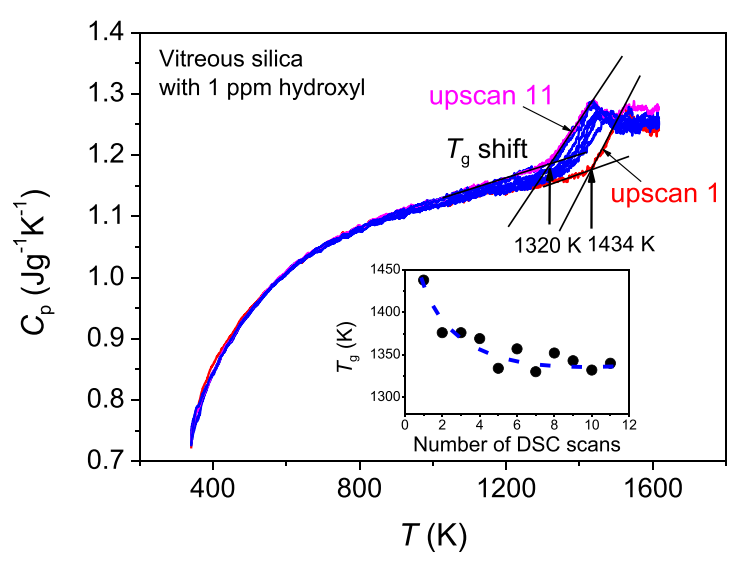

FIGURE 2 | Shift of the onset temperature of glass transition with repeating the DSC up- and downscan at $20 \mathrm{~K} / \mathrm{min}$. The upscan curves 1-11 for the bulk vitreous silica with 1 ppm hydroxyl are shown and the downscan curves are not shown. Inset: the $T_{\mathrm{g}}$ drop trend with increasing the number of DSC scans. The dashed line is guide for eyes.

1982). The viscosity $(\eta)$ - temperature $(T)$ relations for both $\mathrm{GeO}_{2}$ and $\mathrm{CaP}_{2} \mathrm{O}_{6}$ were determined in our previous studies (Yue, 2002; Hornbøll and Yue, 2008b). The $\eta-T$ relation of the basalt system was measured in the present work. The viscosity data in the range of high $T$ were acquired using a concentric cylinder viscometer consisting of a measuring head (Physical Rheolab MC1 Paar Physica) and a high temperature furnace (HT7 Scandia Ovnen), while those at low $T$ (near glass transition) were measured using a micro-penetration viscometer with a vertical dilatometer (Model VIS 405, Bähr-Termoanalyse). The details of both methods can be found elsewhere (Solvang et al., 2004). The viscosity data were fitted to the viscosity model [Eq. (1)] proposed by Mauro et al., (2009b) to determine the liquid fragility index $(m)$ of $\mathrm{SiO}_{2}, \mathrm{GeO}_{2}$, basalt, and $\mathrm{CaP}_{2} \mathrm{O}_{6}$ as shown in Table 1 . 


\section{Sub- $T_{g}$ Annealing}

The annealing experiments were performed on the HQ samples in an electric furnace at various temperatures below $T_{\mathrm{g}}$ under atmospheric atmosphere. The samples were directly put into the furnace at the given annealing temperature $T_{\mathrm{a}}$. This means that the samples underwent a rapid heating to $T_{\mathrm{a}}$. Upon annealing for time $t_{\mathrm{a}}$, the samples were directly taken out from the furnace at $T_{\mathrm{a}}$, giving a cooling rate of about $100 \mathrm{~K} / \mathrm{s}$. Thus, the additional annealing time for the temperature range of $T<T_{\mathrm{a}}$ can be neglected compared to the isothermal annealing time.

\section{Glass Transition Temperature of Vitreous Silica}

It is challenging to determine the glass transition temperature $\left(T_{\mathrm{g}}\right)$ of vitreous silica (Nascimento and Zanotto, 2007). In the present paper, we show the two major factors that cause difficulty in getting the real $T_{\mathrm{g}}$ value of vitreous silica. First, the incorporation of hydroxyl groups into the glass network is difficult to be avoided during production of vitreous silica and it is almost impossible to produce "absolutely dry" vitreous silica. Even 1 ppm hydroxyl could drastically reduce the $T_{\mathrm{g}}$ of vitreous silica. The $T_{\mathrm{g}}$ of $1438 \mathrm{~K}$ of the bulk vitreous silica with $1 \mathrm{ppm}$ $\mathrm{OH}$ (Figure 1) is lower than that of the vitreous silica with $<1$ ppm hydroxyl) reported in the literature, i.e., $1480 \mathrm{~K}$ ) (Richet et al., 1982). To the best of our knowledge, the so-far reported calorimetric $T_{\mathrm{g}}$ values of vitreous silica are lower than $1480 \mathrm{~K}$. This implies that the vitreous silica with $T_{\mathrm{g}}=1480 \mathrm{~K}$ is close to the "perfectly dry" state. Second, the calorimetric standard $T_{\mathrm{g}}$ is determined as the onset temperature of the glass transition peak of the $C_{\mathrm{p}}$ curve obtained from the second DSC upscan at a rate equal to that of the prior downscan rate (Yue, 2008). The thusobtained $T_{\mathrm{g}}$ agrees with the $T_{\mathrm{g}}$ determined using the enthalpymatching method from the second upscan $C_{\mathrm{p}}$ curve (Moynihan et al., 1976; Yue et al., 2002a). However, different from silicate glasses, vitreous silica is rather sensitive to the repeated dynamic heating-cooling cycles in terms of glass structure. During the first DSC up- and downscans, vitreous silica has already gone structure changes due to structural relaxation, and leads to a decrease of $T_{\mathrm{g}}$ (Figure 2). For example, the $T_{\mathrm{g}}$ of the sample subjected to one heating-cooling circle in DSC is lower than that of the as-produced sample.

Figure 1 shows the calorimetric glass transition of vitreous silica, which is characteristic of a much smaller jump in isobaric heat capacity $C_{\mathrm{p}}\left(\Delta C_{\mathrm{p}}=0.07 \mathrm{Jg}^{-1} \mathrm{~K}^{-1}\right)$ from glass to liquid state compared to that of normal oxide glass systems. The small $C_{\mathrm{p}}$ jump indicates that vitreous silica is thermodynamically strong (Angell, 1995). It has been reported that $T_{\mathrm{g}}$ of vitreous silica is $1480 \mathrm{~K}$ (Richet et al., 1982). The striking phenomenon for vitreous silica is that $T_{\mathrm{g}}$ is extremely sensitive to hydroxyl content (Figure 1). The $\mathrm{HQSiO}_{2}$ studied in this work contains 1021 ppm hydroxyl and, hence, has a significantly lower $T_{\mathrm{g}}(1336 \mathrm{~K})$ than that $(1434 \mathrm{~K})$ of the vitreous silica with $1 \mathrm{ppm}$ hydroxyl. Increasing hydroxyl content from 1 to $1021 \mathrm{ppm}$ leads to a drastic increase of nonbridging oxygen fraction, which depolymerizes the structural network of glass, and consequently lowers $T_{\mathrm{g}}$ and viscosity $(\eta)$.
In addition, with increasing hydroxyl content, the $\log \eta \sim 1 / T$ relation of molten silica becomes slightly non-Arrhenian, i.e., the liquid fragility becomes higher than those of $\mathrm{SiO}_{2}$ with $1 \mathrm{ppm}$. Based on the Adam-Gibbs relation (Adam and Gibbs, 1965; Mauro et al., 2009b), the increase in non-Arrhenian degree of relaxation is due to the fact that the configurational entropy $\left(S_{c}\right)$ becomes more dependent on temperature. This means that the incorporation of hydroxyl into the structural network leads to an increase of $S_{\mathrm{c}}$ at a given $T$. In this work, the $\mathrm{HQSiO}_{2}$ glass with 1021 ppm hydroxyl can still be considered to be a strong system, since its viscosity-temperature relation is close to the Arrhenian one (Murach and Brückner, 1997).

To further check whether the $T_{\mathrm{g}}$ decrease of vitreous silica with hydroxyl content is indeed caused by the role of hydroxyl in depolymerizing the structural network, we compare the metallic impurity level between the fiber and bulk samples since the impurities depolymerize the structural network as well. As stated in the experimental section, the bulk sample with $1 \mathrm{ppm}$ hydroxyl contains $\sim<20$ ppm metallic impurity, whereas the fiber sample with $1021 \mathrm{ppm}$ has $<1 \mathrm{ppm}$ impurity. The fiber sample with lower impurity would have a higher $T_{\mathrm{g}}$ than the bulk sample with higher impurity, if the hydroxyl level were the same. Figure 1 shows that the fiber sample has a lower $T_{\mathrm{g}}$ than the bulk sample. This clearly indicates that the hydroxyl content is the governing factor causing the $T_{\mathrm{g}}$ drop displayed in Figure 1.

Figure 2 demonstrates how sensitive the $T_{\mathrm{g}}$ of silica is to the repeated heating protocol, i.e., to the repeated DSC scans (from scan 1 to 11$)$ at the same up- and downscan rate $(20 \mathrm{~K} / \mathrm{min})$. We observe a considerable drop in $T_{\mathrm{g}}$ with repeating the DSC scans. From the $C_{\mathrm{p}}$ curves, it is identified that all the 11 upscan $C_{\mathrm{p}}$ curves overlap with each other below $T_{\mathrm{g}}$, indicating that the vibrational entropy does not vary with the repeating the DSC scan. This implies that the configurational entropy change in the sample from scan 1 to 11 is mainly responsible for the shift of the glass transition peak to lower temperature with repeating DSC scans. In other words, the onset temperature of the jump from vibrational to configurational heat capacity is lowered by repeating the DSC scans. The inset of Figure 2 shows a non-linear $T_{\mathrm{g}}$ decrease with repeating the DSC scan. It is seen that $T_{\mathrm{g}}$ decreases sharply from DSC scan 1 to 2, and afterwards gently falls to a constant value with increasing the DSC scan number. This implies that the structure of vitreous silica may relax to an equilibrium state upon repeated heating-cooling cycles.

Vitreous silica exhibits numerous anomalies, e.g., anomalous thermal expansion, volume relaxation (Brückner, 1971), and liquid-liquid transition (Lascaris et al., 2014). Now we also see the anomalous enthalpy relaxation caused by repeating the heating-cooling circle. Upon the repeated DSC scans, the atomic bonding between structural units $\left(\mathrm{SiO}_{4}\right.$ tetrahedra) becomes looser. The topological origin of the $T_{\mathrm{g}}$ shift is that the topological constraints are weakened by the repeated heating-cooling cycles, and thereby the onset temperature for the constraints to become rigid upon cooling is lowered (Smedskjaer et al., 2010). However, a quantitative description about the topological and structural origin of the $T_{\mathrm{g}}$ shift is missing. To do so, a detailed structural analysis needs to be done in the future work. 
It should be mentioned that there is a tendency for cristobalite to form on the surface of vitreous silica upon DSC upscanning to $1623 \mathrm{~K}$ (Jensen et al., 2009). But this type of surface crystallization should not be the reason for the $T_{\mathrm{g}}$ decrease of silica with increasing the number of upscan. If this were the case, the $C_{\mathrm{p}}$ curve in the temperature range below $T_{\mathrm{g}}$ would decline with repeating the DSC scan. Thus, the internal structure change of vitreous silica, instead of the surface crystallization, must be responsible for the $T_{\mathrm{g}}$ shift to lower temperature. The $T_{\mathrm{g}}$ shift phenomenon of vitreous silica is strongly in contrast to the behavior of other glass formers such as low $T_{\mathrm{g}}$ silicate glasses. For instance, the glass transition peak of low $T_{\mathrm{g}}$ silicate glasses remains unchanged and reproducible with repeating the DSC scan. This means that the structure of vitreous silica is much more sensitive to the repeated heating-cooling cycles compared to normal glass formers.

Now we know that the difficulty in determining the true $T_{\mathrm{g}}$ of vitreous silica lies in the fact that the $T_{\mathrm{g}}$ is extremely sensitive to both the hydroxyl content and the number of the heating-cooling cycles. This remind us that when we adopt the $T_{\mathrm{g}}$ value of vitreous silica from the literature, we should always be aware of the two factors, i.e., the hydroxyl content of the sample and the number of the DSC scans for determining $T_{\mathrm{g}}$. Otherwise, the $T_{\mathrm{g}}$ value would be of high uncertainty for vitreous silica. However, despite the experimental difficulty, we expect that it is still possible to obtain the real $T_{\mathrm{g}}$ of vitreous silica. To do so, it is necessary to do the following experiments in the future work. First, we need to prepare a series of vitreous silica samples covering a large range of hydroxyl contents, measure their $T_{\mathrm{g}}$ values, and then find the relation between $T_{\mathrm{g}}$ and hydroxyl content. Based on this relation, we can extrapolate the $T_{\mathrm{g}}$ trend to the $T_{\mathrm{g}}$ value at 0 hydroxyl content. Second, we need to find the quantitative dependence of the $T_{\mathrm{g}}$ of vitreous silica on the number of the repeated DSC upscan, and then extrapolate it to the 0 scan. The thus-derived $T_{\mathrm{g}}$ can be regarded as the true glass transition temperature of vitreous silica. Implementing the above-mentioned procedures, we expect that the $T_{\mathrm{g}}$ value would approach the true $T_{\mathrm{g}}$ value of dry vitreous silica. An alternative way for determining the standard $T_{\mathrm{g}}$ of vitreous silica is to measure the temperature dependence of viscosity in a large range of temperatures, and thereby to find the temperature corresponding to the viscosity of $10^{12} \mathrm{~Pa}$ s (Yue, 2008). However, it is not straightforward to measure the accurate viscosity data of silica, since rather high temperatures are needed, where chemical disassociation could occur. The viscosity measurements should be conducted within a short period of time.

\section{Enthalpy Relaxation}

Figure 3 illustrates the fragility diagram (Angell, 1995) of the four glass-forming systems. The measured viscosity data were fitted to the following equation that describes the $T_{\mathrm{g}}$-scaled temperature dependence of viscosity (Mauro et al., 2009b):

$$
\begin{aligned}
\log _{10} \eta(T)= & \log _{10} \eta_{\infty}+\left(12-\log _{10} \eta_{\infty}\right) \frac{T_{\mathrm{g}}}{T} \\
& \exp \left[\left(\frac{m}{12-\log _{10} \eta_{\infty}}-1\right)\left(\frac{T_{\mathrm{g}}}{T}-1\right)\right]
\end{aligned}
$$

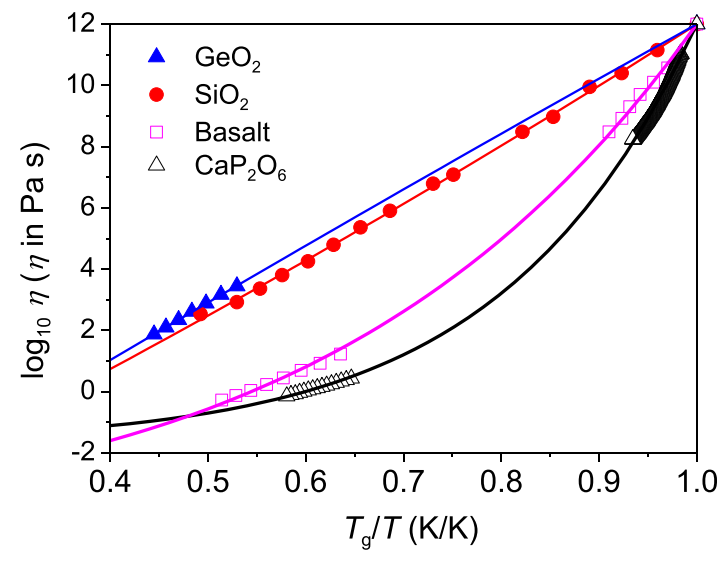

FIGURE 3 | Fragility plots (viscosity $\eta$ versus the $\boldsymbol{T}_{\mathrm{g}}$-scaled temperature $\boldsymbol{T}$ ) of the four systems. Symbols represent the measured viscosity data of $\mathrm{GeO}_{2}$ (Hornbøll and Yue, 2008b), $\mathrm{SiO}_{2}$ (Urbain et al., 1982), basalt (this work), and $\mathrm{CaP}_{2} \mathrm{O}_{6}$ (Yue, 2002). Solid lines show the fits of the measured data to the Eq. (1). The $T_{9}$ values of $\mathrm{GeO}_{2}$, basalt, and $\mathrm{CaP}_{2} \mathrm{O}_{6}$ are given in Table 1, and the $T_{g}$ value of silica (Urbain et al., 1982) is $1406 \mathrm{~K}$. The fragility index $m$ was found to be $18,20,44$, and 69 for $\mathrm{GeO}_{2}, \mathrm{SiO}_{2}$, Basalt, and $\mathrm{CaP}_{2} \mathrm{O}_{6}$, respectively.

where $\eta_{\infty}$ is the high temperature limit of viscosity and $m$ is the fragility index (Angell, 1995). The viscosity at $T_{\mathrm{g}}$ is equal to $10^{12} \mathrm{~Pa}$ s for inorganic glass formers (Yue, 2008, 2009). The $m$ values are listed in Table 1. The solid lines are the fit of the measured data to Eq. (1). It is seen that the fragility index $m$ has the following sequence: $\mathrm{GeO}_{2}<\mathrm{SiO}_{2}<$ Basalt $<\mathrm{Ca}\left(\mathrm{PO}_{3}\right)_{2}$. Here, the question is how the relaxation behavior in HQ glasses relates to their liquid fragility. This will be answered below.

Figures $4 \mathbf{A}, \mathbf{B}$ show the $T_{\mathrm{g}}$ normalized temperature dependences of the isobaric heat capacities $\left(C_{\mathrm{p}}\right)$ for the $\mathrm{HQBas}$ and $\mathrm{HQSiO}_{2}$, respectively, for both the first and the second DSC upscans, i.e., $C_{\mathrm{p} 1}$ and $C_{\mathrm{p} 2}$ curves. The deviation of $C_{\mathrm{p} 1}$ from $C_{\mathrm{p} 2}$, i.e., the broad exothermic peak, indicates that the energy is released from the HQ samples upon dynamic heating (Yue et al., 2002b). The energy release from the samples is an enthalpy recovery process, i.e., a recovery from the enthalpy of HQ glass to that of standard glass. Standard glass refers to the glass subjected to cooling at $20 \mathrm{~K} / \mathrm{min}$, and to subsequent reheating at $20 \mathrm{~K} / \mathrm{min}$. The area embraced by $C_{\mathrm{p} 1}$ and $C_{\mathrm{p} 2}$ is the recovered enthalpy, which is equal to the energy trapped in glass by hyperquenching. The enthalpy relaxation of both $\mathrm{HQBas}$ and $\mathrm{HQSiO}_{2}$ is clearly seen in Figures 4A,D. To analyze the details of the relaxation process, the excess heat capacity, $C_{\mathrm{p}, \mathrm{exc}}=C_{\mathrm{p} 2}-C_{\mathrm{p} 1}$, is plotted against the $T_{\mathrm{g}}$ normalized temperature, $T / T_{\mathrm{g}}$, as shown in Figures $4 \mathrm{C}, \mathrm{D}$. The total excess energy could be estimated by integrating the excess heat capacity $C_{\mathrm{p} \text {,exc }}$ over the entire temperature range of the DSC scan.

Comparing $\mathrm{HQBas}$ with $\mathrm{HQSiO}_{2}$ in Figures 4C,D, we detect two striking differences in the enthalpy relaxation. First, the HQBas exhibits a broader and less symmetrical $C_{\mathrm{p} \text {,exc }}$ peak compared to $\mathrm{HQSiO}_{2}$. This suggests that the distribution of the excitation kinetic energy for HQBas, which induces release of the trapped potential energy, is broader and more complex than for $\mathrm{HQSiO}_{2}$. The difference in the $C_{\mathrm{p} \text {,exc }}$ peak between the two 


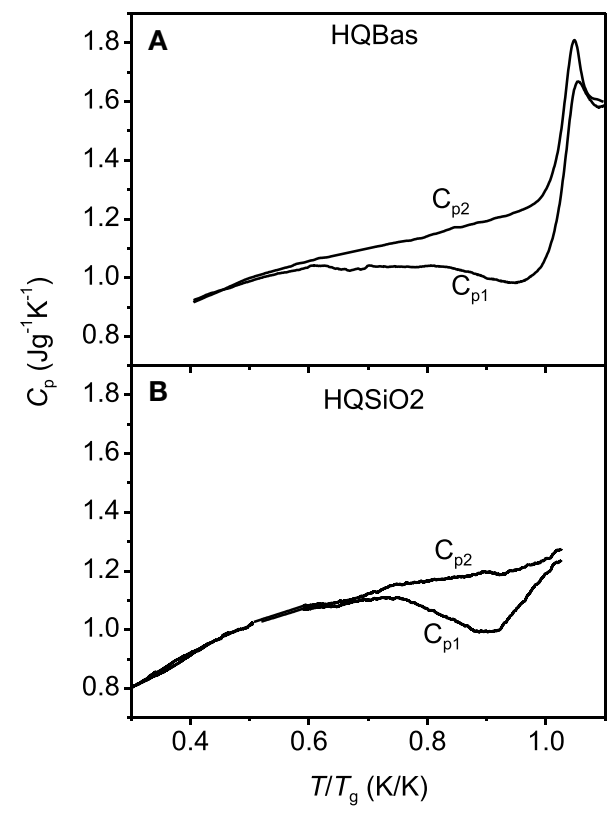

FIGURE 4 | Left panel: Comparison in the isobaric heat capacity $\left(C_{p}\right)$ as a function of the $T_{\mathrm{g}}$-normalized temperature $\left(T / T_{\mathrm{g}}\right)$ between $\mathbf{( A )}$ HQBas (hyperquenched basaltic glass) (Yue et al., 2002a) and (B) $\mathrm{HQSiO}_{2}$ (this work). The $T_{\mathrm{g}}$ values are given in Table 1. $C_{\mathrm{p} 1}$ is the heat capacity of the $\mathrm{HQ}$ samples measured during the first DSC upscan at $20 \mathrm{~K} / \mathrm{min} . C_{\mathrm{p} 2}$ is the heat capacity of the samples relaxed during the first downscan at $20 \mathrm{~K} / \mathrm{min}$ and measured

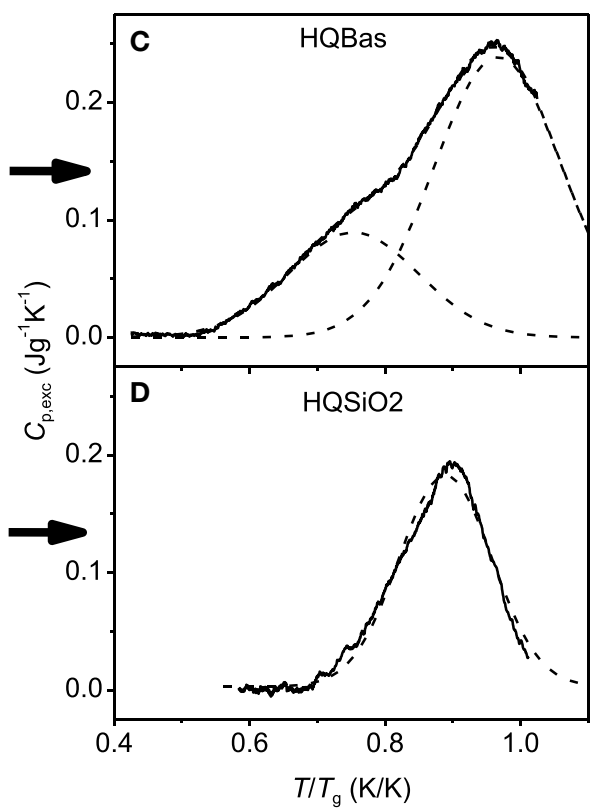

during the second upscan at $20 \mathrm{~K} / \mathrm{min}$. Right panel: Comparison in the excess heat capacity $C_{p, \text { exc }}\left(=C_{p 2}-C_{p 1}\right)$ as a function of $T / T_{\mathrm{g}}$ between

(C) HQBas and (D) $\mathrm{HQSiO}_{2}$. Solid lines represent the measured $C_{\mathrm{p}, \mathrm{exc}}$ curves, while the dashed lines represent the Gaussian fits. The $C_{p, \text { exc }}$ peak of HQBas is the sum of two Gaussian peaks, whereas that of $\mathrm{HQSiO}_{2}$ is described by one Gaussian peak. systems implies that strong glass systems have less the configurational microstates than fragile ones. The structural origin of the difference could be described as follows. The structure of the HQB contains various chemical bonding types such as $\mathrm{Si}-\mathrm{O}$, $\mathrm{Al}-\mathrm{O}, \mathrm{Na}(\mathrm{K})-\mathrm{O}, \mathrm{Ca}(\mathrm{Mg}, \mathrm{Fe})-\mathrm{O}$, whereas that of the $\mathrm{HQSiO}_{2}$ mainly consists of only Si-O bonds but with different potentials. The width of the $C_{\mathrm{p}, \text { exc }}$ peak of the HQ glasses could be associated with the distribution of the chemical bond angle, length, and strength of glass. This distribution determines the overall structural arrangement, and hence the distribution of potential energy in glass. As shown in Figure $4 \mathrm{D}$, the relatively symmetrical $C_{\mathrm{p}, \text { exc }}$ peak of $\mathrm{HQSiO}_{2}$ indicates that one type of chemical bond, i.e., the $\mathrm{Si}-\mathrm{O}$ bonds are involved, but there exists a distribution of the bond angles and bond lengths, and hence a distribution of potential energy, and the latter is narrower than that of multicomponent oxide glasses. The distribution of potential energy in a glass becomes broader with increasing its $T_{\mathrm{f}}$ by raising the cooling rate. A higher $T_{\mathrm{f}}$ glass is subjected to a higher degree of the network distortion. As long as the upscan or annealing time is shorter than the relaxation time, the distorted state of network would remain. When the former is close to or longer than the latter, the distorted network would recover, resulting in an energy release.

Second, the $C_{\text {pexc }}(T)$ peak of the HQBas consists of a "shoulder" and a primary peak (Figure 4C), whereas that of the $\mathrm{HQSiO}_{2}$ consists of only one primary peak (Figure 4D). The relaxation peak of HQBas is deconvoluted into two Gaussian ones in low and high temperature regions, respectively (dashed lines in
Figure 4C). The existence of the "shoulder" suggests that there are two distributions of relaxation time that overlap to some extent in the temperature range below $T_{\mathrm{g}}$. The smaller Gaussian distribution at low temperatures could be associated with relaxation of the bonds between oxygen and modifiers with lower field strength, and also with a higher distortion degree of the oxygenmodifier bonds with higher potential energy. By contrast, the larger primary peak at high temperatures is correlated with relaxation of the bonds between network former and oxygen (i.e., $\mathrm{Si}-\mathrm{O}$ and Al-O bonds). However, the $C_{\text {pexc }}(T)$ peak of $\mathrm{HQSiO}_{2}$ can be described by one Gaussian distribution. This implies that the single relaxation peak of $\mathrm{HQSiO}_{2}$ is associated with only one distribution of relaxation time. This Gaussian distribution could be attributed to the distribution of the Si-O-Si bond angles, leading to a distribution of potential energy. The higher the cooling rate is, the higher is the frozen-in degree of the disorder of the $\mathrm{SiO}_{4}$ network, the broader is the distribution of the bond angles, and the larger is the $C_{\text {pexc }}(T)$ peak.

It has been reported that the potential energy landscape of a strong system may consists of a single metabasin, whereas that of a fragile system displays a proliferation of two wellseparated metabasins (Debenedetti and Stillinger, 2001). From the kinetic fragility diagram (Figure 3) and Table 1, we know that the basaltic system is more fragile than $\mathrm{SiO}_{2}$. Accordingly, the two different distributions of relaxation time of the HQBas could relate to a diversity of deep landscape traps and of the pathways of configurational space that connect them, while the single distribution of relaxation time of the $\mathrm{HQSiO}_{2}$ could be 
linked to a much less diversity of those in the single metabasin of a strong system (Debenedetti and Stillinger, 2001). The silica liquid exhibits almost constant activation energy of $540 \mathrm{~kJ} / \mathrm{mol}$ (calculated from Figure 4). This constancy indicates that the underlying mechanism, e.g., breaking and reformation of $\mathrm{Si}-\mathrm{O}$ bonds, applies throughout the entire landscape. During the sub- $T_{\mathrm{g}}$ annealing, those bonds influence with each other when they break and reform. This is attributed to the high network connectivity of vitreous silica. By contrast, a fragile glass former like basalt exhibits a non-Arrhenius flow behavior, and hence a heterogeneous landscape. This could be the reason for the occurrence of the asymmetrical enthalpy relaxation peak in the basaltic glass (Figure 4D).

Figures 5A,B show the effect of the annealing temperature $T_{\mathrm{a}}$ on the enthalpy relaxation of the two fragile systems: basaltic system and calcium metaphosphate system, respectively. By contrast, Figures 5C,D show the annealing effect on the relaxation of the two strong systems: $\mathrm{SiO}_{2}$ and $\mathrm{GeO}_{2}$, respectively. For the fragile systems, with increasing $T_{\mathrm{a}}$ below $T_{\mathrm{g}}$ for $12 \mathrm{~h}$ the $C_{\mathrm{p}, \text { exc }}$ peak gradually disappears from lower to higher temperature until the entire $C_{\text {pexc }}$ peak vanishes. The left side of the peak shifts to higher temperature, whereas the right side remains unchanged. In comparison, with increasing $\mathrm{T}_{\mathrm{a}}$ (below $T_{\mathrm{g}}$ ) for $24 \mathrm{~h}, \mathrm{HQSiO}_{2}$ gradually releases its excess energy in the entire temperature range that the $C_{\mathrm{p} \text {,exc }}$ peak covers, and this phenomenon manifests itself as a decrease in the peak height. This indicates that a single annealing temperature (representing the kinetic energy) can induce the release of the excessive potential energy of all the excited structural units (e.g., $\mathrm{SiO}_{4}$ tetrahedra) in $\mathrm{HQSiO}_{2}$. Such relaxation feature again implies that there exists only a single metabasin in strong systems (Debenedetti and Stillinger, 2001), which consists of basins with different depths (e.g., different bond energies). The basin depth is distributed over the collective configurational coordinates. A single annealing temperature, i.e., a given single excitation energy $k T_{\mathrm{a}}$ for a given $t_{\mathrm{a}}$, can cause the structural units to relax successively by sampling basins from shallow to deep ones. Increasing $T_{\mathrm{a}}$ for a given $t_{\mathrm{a}}$ leads to an increase in the degree of relaxation by sampling deeper basins. The energy release of $\mathrm{HQSiO}_{2}$ at any $T_{\mathrm{a}}$ (below $T_{\mathrm{g}}$ ) is accompanied by the relaxation of the hyperquenching excited $\mathrm{Si}-\mathrm{O}$ bonds upon sub- $T_{\mathrm{g}}$ annealing. Driven by the thermal kinetic energy, the $\mathrm{SiO}_{4}$ tetrahedra and the rings consisting of $\mathrm{SiO}_{4}$ tetrahedra collaboratively adjust themselves from their original positions to more stable position. During this process, these structural units are still connected with each other and therefore are influenced by each other. A similar enthalpy relaxation mode is also observed in $\mathrm{HQGeO}_{2}$, indicating that the cooperative relaxation is a universal feature of strong glass formers. The Johari-Goldstein (JG) relaxation has been detected in $\mathrm{HQGeO}_{2}$ by using the hyperquenchingannealing-calorimetric scanning approach although the JG relaxation is difficult to be isolated from the primary relaxation (Hu and Yue, 2008).

Figures 5A,B could indicate the relaxation of the fragile systems during annealing at a given temperature proceeds in the manner that the excess energy release first starts from highly untestable structural domains and ends in the slightly unstable ones. This relaxation feature can be explained in terms of the energy landscape of fragile systems as mentioned above. The difference in relaxation behavior between strong and fragile systems also indicates that the energetic and structural heterogeneities in both $\mathrm{HQBas}$ and $\mathrm{HQCmP}$ at a given $T_{\mathrm{f}}$ are higher than those of $\mathrm{HQSiO}_{2}$ and $\mathrm{HQGeO}_{2}$ (Yue and Angell, 2004).

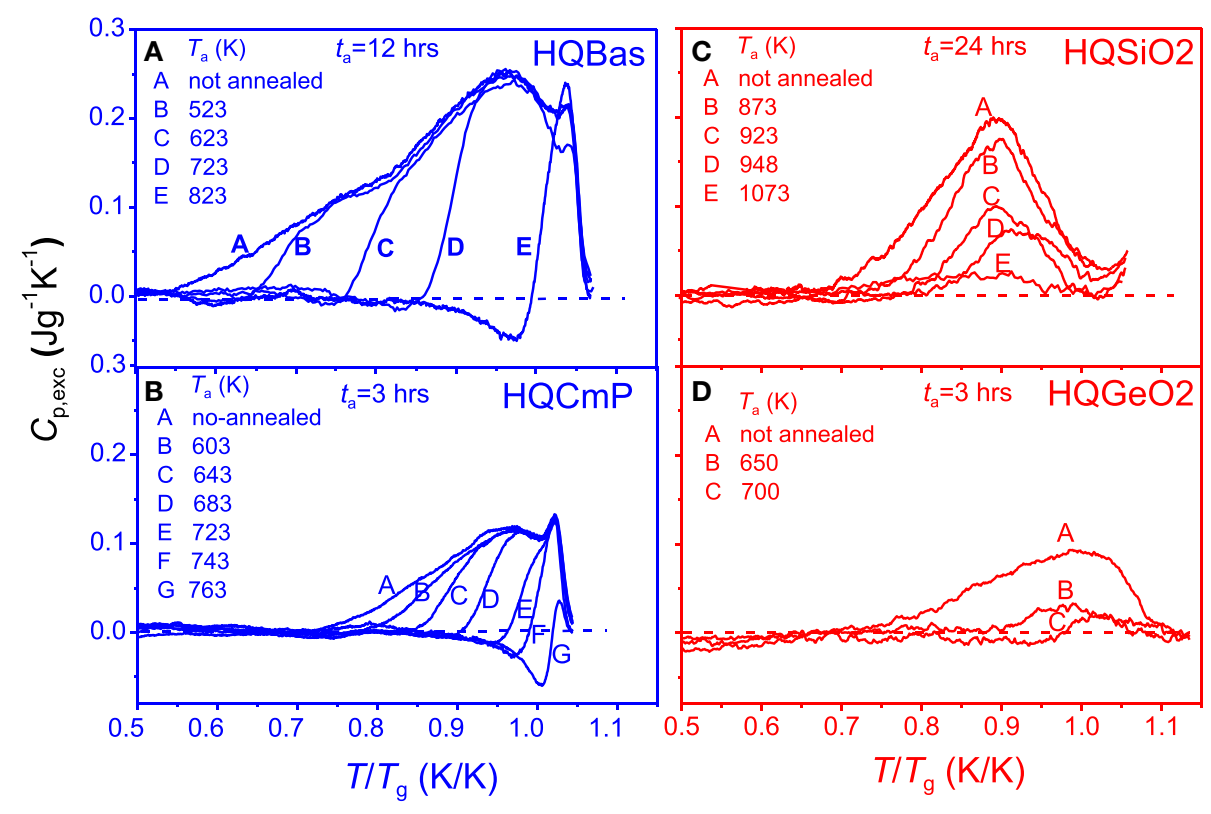

FIGURE 5 | Effect of the annealing temperature $T_{\mathrm{a}}$ on the $\boldsymbol{C}_{\mathrm{p}, \mathrm{exc}}$ as a function of $\boldsymbol{T} / \boldsymbol{T}_{\mathrm{g}}$ for a given annealing duration $\boldsymbol{t}_{\mathrm{a}}$. The $T_{\mathrm{g}}$ values are given in Table 1. Left panel (fragile systems): (A) HQBas (Yue et al., 2002a); (B) HQCmP (Yue, 2002). Right panel (strong systems): (C) $\mathrm{HQSiO}_{2}$ containing 1021 ppm hydroxyl (this work); (D) $\mathrm{HQGeO}_{2}$ (Hornbøll and Yue, 2008b). The dashed lines represent the $C_{p, e x c}$ peak (as reference) obtained on the samples cooled at $20 \mathrm{~K} / \mathrm{min}$. 
In addition, Figures 5A-D exhibit another pronounced difference in the enthalpy recovery between the fragile systems and strong systems. In the annealed HQBas and HQCmP glasses, an endothermic pre-peak (i.e., the peaks under zero lines of $\left.C_{\mathrm{p} \text {,exc }}\right)$ is observed prior to the exothermic energy release peak, when $T_{\mathrm{a}}$ is sufficiently high (but below $T_{\mathrm{g}}$ ). The extent of the pre-peak can be enhanced by raising $T_{\mathrm{a}}$. Such a pre-peak is a consequence of the non-exponentiality of enthalpy relaxation, reflecting the energy heterogeneity in HQ fragile glass samples (Yue and Angell, 2004). However, the pre-peak is not observed in $\mathrm{HQSiO}_{2}$ and $\mathrm{HQGeO}_{2}$ (see Figures 5C,D). The lack of the pre-peak in the $C_{\text {p,exc }}$ spectra is another evidence for the lower energetic and structural heterogeneities in strong glass formers.

Here, it is important to point out that for a comparable cooling rate the $T_{\mathrm{f}} / T_{\mathrm{g}}$ ratios of the HQ strong glass systems $\left(\mathrm{HQSiO}_{2}\right.$ and $\mathrm{HQGeO}_{2}$ ) are significantly higher than those of the HQ fragile ones (HQBas and HQCmP) as shown in Table 1. This is expected since a strong liquid have a much smaller configurational heat capacity than a fragile one. In other words, compared to a fragile system, a strong one undergoes a smaller configurational entropy jump when its glass state is transformed into a metastable liquid state. This is due to the fact that the structural difference in both short-range order and medium-range order between glass and liquid at $T_{\mathrm{g}}$ is smaller for a strong system than for a fragile one. As a consequence, a similar amount of configurational enthalpy should be trapped at a higher $T_{\mathrm{f}} / T_{\mathrm{g}}$ ratio for a strong system than a fragile one when both are quenched at the same rate if the enthalpy-matching method for determining both the $T_{\mathrm{g}}$ and the $T_{\mathrm{f}}$ of HQ glasses is used (Moynihan et al., 1976; Yue et al., 2002a). In addition, the structural rearrangement in strong liquids during cooling is rather sluggish and, hence, is more readily frozen-in at a higher $T_{\mathrm{g}}$-normalized temperature compared to fragile ones.

In Figures 6A-D, the $C_{\mathrm{p}, \text { exc }}$ is plotted against $T / T_{\mathrm{g}}$ for the four HQ glasses, showing the effect of $t_{\mathrm{a}}$ on the enthalpy relaxation at a comparable $T_{\mathrm{a}} / T_{\mathrm{g}}$ ratio. Similar to the $T_{\mathrm{a}}$ effect, an increase of $t_{\mathrm{a}}$ results in the shift of the $C_{\mathrm{p} \text {,exc }}$ peak from lower to higher temperature for both HQBas and HQCmP (Figures 6A,B), whereas it causes gradual flattening of $C_{\text {pexc }}$ peak for both $\mathrm{HQSiO}_{2}$ and $\mathrm{HQGeiO}_{2}$ (Figures 6C,D). This indicates that the way of configurational sampling within the potential energy landscape of the strong systems differs from that of fragile ones. For HQBas and HQCmP, at a fixed annealing temperature, i.e., fixed kinetic energy, extending $t_{\mathrm{a}}$ increases the probability for a certain type of the chemical bonds (e.g., modifier-oxygen bonds) to escape from higher to lower basins within the potential energy landscape, until all the bonds of the same type fall below the energy level corresponding to $T_{\mathrm{a}}$. When a basin is sampled, its neighbored basin is influenced and sampled. In other words, the neighbored basins are sampled one after another. During annealing, highly distorted modifier-oxygen ions in HQBas and HQCmP are gradually restored, whereas the distorted bonds remain unaffected. This implies that the structural relaxation of fragile systems is of more heterogeneous nature. By contrast, upon annealing, the relaxation in $\mathrm{HQSiO}_{2}$ is of more cooperative nature. The cooperative relaxation is associated with the coupled rotations of $\mathrm{SiO}_{4}$ tetrahedra (Buchenau et al., 1984) and interaction between
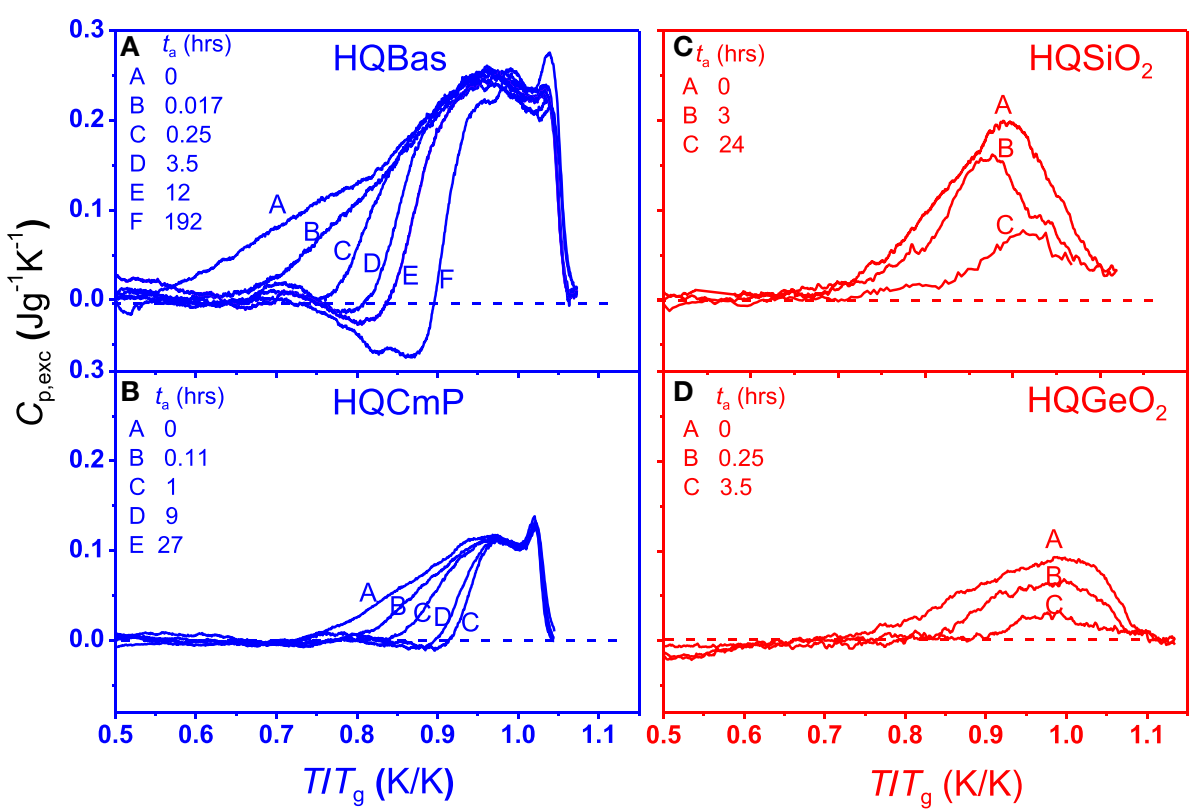

FIGURE 6 | Effect of annealing time ( $\left.\boldsymbol{t}_{\mathrm{a}}\right)$ on $\boldsymbol{C}_{\mathrm{p}, \mathrm{exc}}$ of HQ glasses as a function of $\boldsymbol{T} / \boldsymbol{T}_{\mathrm{g}}$ at a given annealing temperatures $\left(\boldsymbol{T}_{\mathrm{a}}\right)$. The $T_{\mathrm{g}}$ values are given in Table 1. Left panel (fragile systems): (A) HQBas annealed at $T_{\mathrm{a}}=723 \mathrm{~K}\left(0.77 T_{\mathrm{g}}\right.$ ) (Yue et al., 2002a); (B) HQCmP annealed at $T_{\mathrm{a}}=614 \mathrm{~K}\left(0.77 T_{\mathrm{g}}\right.$ ) (Yue, 2002). Right panel (strong systems): (C) $\mathrm{HQSiO}_{2}$ annealed at $T_{\mathrm{a}}=973 \mathrm{~K}\left(0.75 T_{\mathrm{g}}\right)$ (this work); (D) $\mathrm{HQGeO}_{2}$ annealed at $T_{\mathrm{a}}=650 \mathrm{~K}\left(0.78 T_{\mathrm{g}}\right)(\mathrm{Hornb} \varnothing \mathrm{ll}$ and $\mathrm{Yue}, 2008 \mathrm{~b})$ 
different bonds (i.e., bonds with different potentials) due to the high network connectivity of vitreous silica. At a single $T_{a}$ the coupled motion of bonds from non-equilibrated or heavily distorted state to relatively equilibrated state causes relaxation of the entire network structure. That is why the relaxation of the $\mathrm{HQSiO}_{2}$ takes place in the entire temperature range that the $C_{\mathrm{p}, \mathrm{exc}}$ peak covers.

The relaxation of local structural units takes place between the basins via breaking and reforming of bonds. Different local minima can be identified as different bonding types and then energy of minimum coincides with the potential energy of the configuration, i.e., with the number of bonds (Moreno et al., 2005). The structural change caused by extending $t_{\mathrm{a}}$ is a kinetic process, during which a gradual increase occurs in population of the basic structural units that participate in jumping from higher to lower minima. Such jumps are dependent on each other.

Furthermore, at the comparable annealing degree (i.e., similar $T_{\mathrm{a}} / T_{\mathrm{g}}$ ) the pre-peaks appear in HQBas and HQCmP, whereas they are not present in $\mathrm{HQSiO}_{2}$ and $\mathrm{HQGeO}_{2}$ (Figures 5A-D6A-D). This confirms again that during annealing the structural relaxation is of more cooperative nature in the strong system compared to the fragile systems. The finding of such features is of both scientific importance and technological importance. The optical and mechanical properties of $\mathrm{SiO}_{2}$ fibers are strongly influenced by their thermal history (e.g., cooling rate and the degree of annealing), the repeated heating-cooling cycles and mechanical history (e.g., drawing stress) (Martin et al., 2005; Hornbøll et al., 2006; Ya et al., 2008). The outcome of this work is useful for optimizing both forming conditions and annealing process of vitreous silica fibers with respect to their optical and mechanical performances.

Here, it should be noted that, during sub- $T_{\mathrm{g}}$ annealing, not only bulk enthalpy relaxation takes place in glass fibers, but also other types of relaxation proceed, e.g., surface relaxation and axial drawing stress relaxation. According to previous studies (Agarwal and Tomozawa, 1997; Koike and Tomozawa, 2006), the surface structural relaxation of silica glass fiber is faster than that of the silica glass plate. This means that the so-called size effect occurs regarding the surface structural relaxation kinetics, i.e., smaller sized samples exhibit faster relaxation kinetics (Koike and Tomozawa, 2006). The size effect was also found for the tensile strength of glass fibers (Lund and Yue, 2010). The surface structural relaxation of vitreous silica may be the mechanism of the mechanical fatigue (Tomozawa and Hepburn, 2004). However, the enthalpy relaxation of HQ glasses during annealing is a bulk effect, not a surface effect and this is confirmed by the matching between experimental observations and physical modeling of the bulk relaxation (Hornbøll et al., 2010). The effect of the surface absorbed water on the enthalpy relaxation pattern could be ruled out since similar relaxation patterns are also observed in HQ metallic glasses that do not have chemically bonded surface water (Hu et al., 2011). Furthermore, the enthalpy enhancement in glass fibers induced by mechanical stretching is much weaker than that induced by hyperquenching, and this is verified by the sub- $T_{\mathrm{g}}$ relaxation studies (Martin et al., 2005;
Hornbøll et al., 2006). All this implies that the enthalpy relaxation peaks of $\mathrm{HQSiO}_{2}$ (Figures 5C and 6C) arise mainly from the release of the excessive enthalpy trapped in the samples by hyperquenching, but neither from surface relaxation nor from the stretching stress relaxation. This is also the case for $\mathrm{HQ} \mathrm{GeO}_{2}$ (Figures 5D and 6D). Thus, the anomalous enthalpy relaxation in $\mathrm{HQSiO}_{2}$ is a general feature of the HQ strong glass formers. The results of this work have also indirectly confirmed the finding of Mauro and Loucks (2008) that fragile systems have a greater free energy barriers for structural relaxation compared to strong systems. However, a more quantitative study about the relation between enthalpy relaxation and energy landscape need to be carried out in the future.

\section{Conclusion}

$T_{\mathrm{g}}$ of vitreous silica is extremely sensitive to both the hydroxyl content and the repeated heating-cooling cycles. This is why a generally accepted $T_{\mathrm{g}}$ value for silica, based on DSC experiments, is still missing in the literature. We suggest that the true $T_{\mathrm{g}}$ value of vitreous silica should be obtained by extrapolating the $T_{\mathrm{g}} \sim$ hydroxyl content relation to $0 \mathrm{ppm}$, and by extrapolating the repeated DSC scans to 0 scan.

In comparison to fragile oxide systems, the prototypical strong system - HQ vitreous silica exhibit several striking anomalies in enthalpy relaxation. First, the excess heat capacity peak $\left(C_{\mathrm{p}, \text { exc }}\right)$ of the HQ strong systems can be described by a single Gaussian pattern, whereas that of the HQ fragile systems can be deconvoluted into two Gaussian patterns at low and high temperatures, respectively. Second, the excess enthalpy trapped in fragile glasses by hyperquenching is successively released with $T_{\mathrm{a}}$, whereas that of the HQ strong glasses is simultaneously released in the entire temperature range below $T_{\mathrm{g}}$. Third, an endothermic pre-peak appears in the HQ fragile systems, whereas it does not appear in the HQ strong systems. The $\mathrm{HQ} \mathrm{GeO}_{2}$ also shows such anomalies in enthalpy relaxation. This suggests that those anomalies in enthalpy relaxation are a universal feature of strong glass formers. These anomalies could be qualitatively explained in terms of the potential energy landscape, and they reflect a significant difference in structural rearrangement between the strong and fragile systems. The anomalies also imply that the bond relaxations of different types of bonds in HQ fragile systems are of independent nature, while those in HQ strong systems are of cooperative nature. Fragile systems possess a higher degree of energetic and structural heterogeneity than the strong ones. With increasing the annealing temperature and time, the excess enthalpy of the HQ vitreous silica relaxes throughout the entire energy landscape.

\section{Acknowledgments}

This work was financially supported by Danish Research Council under the Grant no. 26-03-0096. The authors would like to thank J. Murach for providing vitreous silica fibers and R. Müller for measuring hydroxyl content in vitreous silica fibers. 


\section{References}

Adam, G., and Gibbs, J. H. (1965). On the temperature dependence of cooperative relaxation properties in glass forming liquids. J. Chem. Phys. 43, 139-146. doi:10.1063/1.1696442

Agarwal, A., and Tomozawa, M. (1997). Surface and bulk structural relaxation kinetics of silica glass. J. Non Cryst. Solids 209, 264-272. doi:10.1016/ S0022-3093(96)00570-4

Angell, C. A. (1995). Formation of glasses from liquids and biopolymers. Science 267, 1924-1935. doi:10.1126/science.267.5206.1924

Angell, C. A., Yue, Y. Z., Wang, L. M., Copley, J. R. D., Borick, S., and Mossa, S. (2003). Potential energy, relaxation, vibrational dynamics and the boson peak, of hyperquenched glasses. J. Phys. Condens. Matter 15, S1051-S1068. doi:10.1088/0953-8984/15/11/327

Bateson, S. (1958). Critical study of the optical and mechanical properties of glass fibers. J. Appl. Phys. 29, 13. doi:10.1063/1.1722934

Brückner, R. (1971). Properties and structure of vitreous silica. II. J. Non Cryst. Solids 5, 177-216. doi:10.1016/0022-3093(71)90032-9

Buchenau, U., Nücker, N., and Dianoux, A. J. (1984). Neutron scattering study of the low-frequency vibrations in vitreous silica. Phys. Rev. Lett. 53, 2316. doi:10.1103/PhysRevLett.53.2316

Chumakov, A. I., Monaco, G., Fontana, A., Bosak, A., Hermann, R. P., Bessas, D., et al. (2014). Role of disorder in the thermodynamics and atomic dynamics of glasses. Phys. Rev. Lett. 112, 025502. doi:10.1103/PhysRevLett.112.025502

Debenedetti, P. G., and Stillinger, F. H. (2001). Supercooled liquids and the glass transition. Nature 410, 259-267. doi:10.1038/35065704

Deubener, J., Müller, R., Behrens, H., and Heide, G. (2003). Water and the glass transition temperature of silicate melts. J. Non Cryst. Solids 330, 268-273. doi:10.1016/S0022-3093(03)00472-1

Doremus, R. H. (2002). Viscosity of silica. J. Appl. Phys. 92, 7619. doi:10.1063/1.1515132

Guo, X. J., Mauro, J. C., Potuzak, M., and Yue, Y. Z. (2012). Structural relaxation in annealed hyperquenched basaltic glasses: Insights from calorimetry. J. Non Cryst. Solids 358, 1356-1361. doi:10.1016/j.jnoncrysol.2012.03.009

Hornbøll, L., Knudsen, T., Yue, Y. Z., and Guo, X. J. (2010). Heterogeneous enthalpy relaxation in glasses far from equilibrium. Chem. Phys. Lett. 494, 37-40. doi:10.1016/j.cplett.2010.05.077

Hornbøll, L., Lonnroth, N., and Yue, Y. Z. (2006). Energy release in isothermally stretched silicate glass fibers. J. Am. Ceram. Soc. 89, 70-74. doi:10.1111/j.1551-2916.2005.00736.x

Hornbøll, L., and Yue, Y. Z. (2008a). Enthalpy relaxation of hyperquenched glasses and its possible link to $\alpha$ and $\beta$-relaxations. J. Non Cryst. Solids 354, 350-354. doi:10.1016/j.jnoncrysol.2007.06.101

Hornbøll, L., and Yue, Y. Z. (2008b). Enthalpy relaxation in hyperquenched glasses of different fragility. J. Non Cryst. Solids 354, 1862-1870. doi:10.1016/j. jnoncrysol.2007.10.023

Hu, L. N., and Yue, Y. Z. (2008). Secondary relaxation behavior in a strong glass. J. Phys. Chem. B 112, 9053-9057. doi:10.1021/jp711696p

Hu, L. N., Yue, Y. Z., and Zhang, C. Z. (2011). Abnormal sub- $T_{\mathrm{g}}$ enthalpy relaxation in the CuZrAl metallic glasses far from equilibrium. Appl. Phys. Lett. 98, 081904. doi:10.1063/1.3556659

Huang, J., and Gupta, P. K. (1992). Enthalpy relaxation in thin glass fibers. J. Non Cryst. Solids 151, 175-181. doi:10.1016/0022-3093(92)90026-G

Jensen, M., Keding, R., Höche, T., and Yue, Y. Z. (2009). Biologically formed mesoporous amorphous silica. J. Am. Chem. Soc. 131, 2717-2721. doi:10.1021/ ja808847y

Kiczenski, T. J., and Stebbins, J. F. (2006). The development of a rapid quenching device for the study of the dependence of glass structure on fictive temperature. Rev. Sci. Instrum. 77, 013901. doi:10.1063/1.2162751

Koike, A., and Tomozawa, M. (2006). Size effect on surface structural relaxation kinetics of silica glass sample. J. Non Cryst. Solids 352, 3787-3793. doi:10.1016/j. jnoncrysol.2006.06.020

Lascaris, E., Hemmati, M., Sergey, V., Buldyrev, S. V., Stanley, H. E., and Angell, C. A. (2014). Search for a liquid-liquid critical point in models of silica. J. Chem. Phys. 140, 224502. doi:10.1063/1.4879057
Lund, M. D., and Yue, Y. Z. (2010). Impact of drawing stress on the tensile strength of oxide glass fibers. J.Am. Ceram. Soc. 93, 3236-3243. doi:10.1111/j.1551-2916.2010.03879.x

Martin, B., Wondraczek, L., Deubener, J., and Yue, Y. Z. (2005). Mechanically induced excess enthalpy in inorganic glasses. Appl. Phys. Lett. 86, 121917. doi:10.1063/1.1895483

Mauro, J. C., and Loucks, R. J. (2008). Impact of fragility on enthalpy relaxation in glass. Phys. Rev. E 78, 021502. doi:10.1103/PhysRevE.78.021502

Mauro, J. C., Allan, D. C., and Potuzak, M. (2009a). Nonequilibrium viscosity of glass. Phys. Rev. B 80, 094204. doi:10.1103/PhysRevB.80.094204

Mauro, J. C., Yue, Y. Z., Ellison, A. J., Gupta, P. K., and Allan, D. C. (2009b). Viscosity of glass-forming liquids. Proc. Natl. Acad. Sci. U.S.A. 106, 19780-19784. doi:10.1073/pnas.0911705106

Mei, Q., Benmore, C. J., and Weber, J. K. R. (2007). Structure of liquid $\mathrm{SiO}_{2}$ : a measurement by high-energy x-ray diffraction. Phys. Rev. Lett. 98, 057802. doi:10.1103/PhysRevLett.98.057802

Monaco, A., Chumakov, A. I., Yue, Y. Z., Monaco, G., Comez, L., Fioretto, D., et al. (2006). Density of vibrational states of a hyperquenched glass. Phys. Rev. Lett. 96, 205502. doi:10.1103/PhysRevLett.96.205502

Moreno, A. J., Buldyrev, S. V., La Nave, E., Saika-Voivod, I., Sciortino, F., Tartaglia, P., et al. (2005). Energy landscape of a simple model for strong liquids. Phys. Rev. Lett. 95, 157802. doi:10.1103/PhysRevLett.95.157802

Moynihan, C. T., Easteal, A. J., Debolt, M. A., and Tucker, J. (1976). Dependence of the fictive temperature of glass on cooling rate. J. Am. Ceram. Soc. 59, 12-16. doi:10.1111/j.1151-2916.1976.tb09376.x

Murach, J., and Brückner, R. (1997). Preparation and structure-sensitive investigations on silica glass fibers. J. Non Cryst. Solids 211, 250-261. doi:10.1016/ S0022-3093(96)00635-7

Nascimento, M. L. F., and Zanotto, E. D. (2007). Diffusion processes in vitreous silica revisited. Phys. Chem. Glasses Eur. J. Glass. Sci. Technol. B 48, 201-217.

Richet,P., Bottinga,Y.,Denielou,L.,Petitet,J.P., and Tequi,C.(1982).Thermodynamic properties of quartz, cristobalite and amorphous $\mathrm{SiO}_{2}$ : drop calorimetry measurements between 1000 and $1800 \mathrm{~K}$ and a review from 0 to $2000 \mathrm{~K}$. Geochim. Cosmochim. Acta 46, 2639-2658. doi:10.1016/0016-7037(82)90383-0

Saika-Voivod, I., Poole, P. H., and Sciortino, F. (2001). Fragile-to-strong transition and polyamorphism in the energy landscape of liquid silica. Nature 412, 514-517. doi: $10.1038 / 35087524$

Smedskjaer, M. M., Mauro, J. C., Sen, S., and Yue, Y. Z. (2010). Quantitative design of glassy materials using temperature-dependent constraint theory. Chem. Mater. 22, 5358-5365. doi:10.1021/cm1016799

Solvang, M., Yue, Y. Z., Jensen, S. L., and Dingwell, D. B. (2004). Rheological and thermodynamic behaviors of different calcium aluminosilicate melts with the same non-bridging oxygen content. J. Non Cryst. Solids 336, 179-188. doi:10.1016/j.jnoncrysol.2004.02.009

Thomas, W. F. (1958). Strength of glass fibres. Nature 181, 1006-1006. doi:10.1038/1811006a0

Tomozawa, M., and Hepburn, R. W. (2004). Surface structural relaxation: a possible mechanism of mechanical fatigue. J. Non Cryst. Solids 345, 449-460. doi:10.1016/j.jnoncrysol.2004.08.065

Urbain, G., Bottinga, Y., and Richet, P. (1982). Viscosity of liquid silica, silicates and alumino-silicates. Geochim. Cosmochim. Acta 46, 1061-1072. doi:10.1016/0016-7037(82)90059-X

Ya, M., Deubener, J., and Yue, Y. Z. (2008). Enthalpy and anisotropy relaxation of glass fibers". J. Am. Ceram. Soc. 91, 745-752. doi:10.1111/j.1551-2916.2007.02100.x

Yue, Y. Z. (2002). Fragility of a calcium phosphate melt and relaxation of its glass fibers. Phosphorus Res. Bull. 13, 39-49. doi:10.3363/prb1992.13.0_39

Yue, Y. Z. (2004). Influence of physical ageing on the excessive heat capacity of hyperquenched silicate glass fibers. J. Non Cryst. Solids 348, 72-77. doi:10.1016/j. jnoncrysol.2004.08.128

Yue, Y. Z. (2008). Characteristic temperatures of enthalpy relaxation in glass. J. Non Cryst. Solids 354, 1112-1118. doi:10.1016/j.jnoncrysol.2006.11.027

Yue, Y. Z. (2009). The iso-structural viscosity, configurational entropy and fragility of oxide liquids. J. Non Cryst. Solids 355, 737-744. doi:10.1016/j. jnoncrysol.2009.01.032 
Yue, Y. Z., and Angell, C. A. (2004). Clarifying the glass-transition behaviour of water by comparison with hyperquenched inorganic glasses. Nature 427, 717-720. doi:10.1038/nature02295

Yue, Y. Z., Jensen, S. L., and Christiansen, J. deC. (2002a). Physical aging in a hyperquenched glass. Appl. Phys. Lett. 81, 2983-2985. doi:10.1063/1.1514386

Yue, Y. Z., Christiansen, J. deC., and Jensen, S. L. (2002b). Determination of the fictive temperature for a hyperquenched glass. Chem. Phys. Lett. 357, 20-24. doi:10.1016/S0009-2614(02)00434-7

Yue, Y.Z., von der Ohe, R., and Jensen, S. L. (2004). Fictive temperature, cooling rate and viscosity of glasses. J. Chem. Phys. 120, 8053-8059. doi:10.1063/1.1689951

Zhang, Y. F., Hu, L. N., Liu, S. J., Zhu, C. F., and Yue, Y. Z. (2013a). Sub-Tg enthalpy relaxation in an extremely unstable oxide glass and its implication for structural heterogeneity. J. Non Cryst. Solids 381, 23-28. doi:10.1016/j. jnoncrysol.2013.09.018

Zhang, Y. F., Yang, G., and Yue, Y. Z. (2013b). Calorimetric signature of structural heterogeneity in a ternary silicate glass. J. Am. Ceram. Soc. 96, 3035-3037. doi:10.1111/jace.12562
Zhang, Y. F., Guo, X. J., and Yue, Y. Z. (2013c). Effect of the initial stage of annealing on modeling of enthalpy relaxation in a hyperquenched glass. J. Non Cryst. Solids 378, 121-125. doi:10.1016/j.jnoncrysol.2013.06.029

Zhou, C., Hu, L. N., Sun, Q. J., Zheng, H. J., Zhang, C. Z., and Yue, Y. Z. (2015). Structural evolution during fragile-to-strong transition in $\mathrm{CuZr}(\mathrm{Al})$ glass-forming liquids. J. Chem. Phys. 142, 064508. doi:10.1063/1.4907374

Conflict of Interest Statement: The author declares that the research was conducted in the absence of any commercial or financial relationships that could be construed as a potential conflict of interest.

Copyright (C) 2015 Yue. This is an open-access article distributed under the terms of the Creative Commons Attribution License (CC BY). The use, distribution or reproduction in other forums is permitted, provided the original author(s) or licensor are credited and that the original publication in this journal is cited, in accordance with accepted academic practice. No use, distribution or reproduction is permitted which does not comply with these terms. 